\title{
EXPLORANDO LOS SOCIAL MEDIA EN LA GESTIÓN DE LAS RELACIONES CON LOS CLIENTES EN EMPRESAS TURÍSTICAS
}

\author{
CARMEN DOMÍNGUEZ-FALCÓN ${ }^{1}$ \\ Departamento de Economía y Dirección de Empresa \\ Facultad de Economía, Empresa y Turismos \\ Universidad de Las Palmas de Gran Canaria \\ MARTA CERDÁN-BES ${ }^{2}$ \\ Master's degree in Marketing \\ Universidad Carlos III
}

\begin{abstract}
Resumen: La gestión de las relaciones con los clientes, a través del uso de entornos digitales colaborativos, ha dado lugar al CRM social (SCRM). El SCRM surge como una nueva estrategia de negocio que, a través del uso de plataformas 2.0, va a gestionar de forma más efectiva al cliente al ser capaz de escuchar, satisfacer e incluso anticiparse a cualquier necesidad que requiera, con el fin de mantener relaciones a largo plazo y fidelizarlo. Esta investigación explora la importancia del SCRM en empresas vinculadas al turismo en Gran Canaria (Islas Canarias, España). Para ello, se realizó una investigación exploratoria a través de entrevistas en profundidad a directivos de importantes empresas del sector. Los resultados señalan que se trata de un tema de gran interés y novedad, aunque escasamente implantado de forma estratégica y operativa entre las empresas turísticas. Conocer su impacto en los resultados organizativos supone un reto para la investigación y la práctica empresarial.
\end{abstract}

Palabras claves: Customer Relationship Management (CRM), Social Customer Relationship Management (SCRM), social media, fidelización, turismo.

\section{EXPLORING SOCIAL MEDIA IN THE CUSTOMER RELATIONSHIP MANAGEMENT IN TOURIST COMPANIES}

\begin{abstract}
Managing customer relationships through collaborative digital environments has resulted in social CRM (SCRM). The SCRM emerges as a new business strategy that use 2.0 platforms. Doing so, organization will better manage their customers to be able to listen, meet and anticipate any need that may be required in order to maintain long-term relationships and loyalty. This research explores the importance of SCRM in companies linked to tourism in Gran Canaria (Canary Islands, Spain). For that, an exploratory investigation was carried out through in-depth interviews with executives of leading companies in this sector. The results indicate that SCRM is an issue of great interest and novelty, although scarcely implemented in a strategic and operational way among tourist companies. Knowing its impact on organizational performance is a challenge for research and for practice.
\end{abstract}

Key words: Customer Relationship Management (CRM), Social Customer Relationship Management (SCRM), social media, loyalty, tourism.

\footnotetext{
${ }^{1}$ carmen.dominguez@ulpgc.es

$2100373038 @$ alumnos.uc3m.es
} 


\section{INTRODUCCIÓN}

Las empresas de servicios, especialmente aquellas que pertenecen al sector turístico, desarrollan un gran interés en realizar una gestión efectiva de sus clientes (Maggon y Chaudhry, 2015). Comprender sus necesidades y ofrecerles servicios que añadan valor son factores que van a permitir determinar el éxito o fracaso de estas organizaciones (King y Burgess, 2008; Liao et al., 2010). Esto es, orientarse al cliente posibilita a las empresas de servicios poder superar el incremento de la competencia, así como los efectos de la globalización, y maximizar los ingresos de manera eficiente (Özgener y Iraz, 2006). Con la ayuda de los social media, estas empresas pueden crear vínculos a largo plazo con los usuarios, además de recopilar información valiosa sobre sus necesidades y diferenciarse de la competencia (Cambra-Fierro et al.,2014). Por tanto, los social media han pasado a considerarse una herramienta idónea para lograr una comunicación eficaz, ya que permite a la empresa personalizar el mensaje que envía a los distintos segmentos de clientes a los que se dirige, además de facilitar la interactuación entre ellos (empresa-cliente y cliente-cliente) (Kaplan y Haenlein, 2010; Hollebeek et al., 2014). Al mismo tiempo, el uso estratégico de los social media posibilita que las empresas establezcan un vínculo positivo hacia la marca con la que el cliente puede sentirse identificado (Palazón et al., 2014). De este modo, surge el novedoso concepto de Social Customer Relationship Management o Social CRM (SCRM), como una evolución de la gestión de las relaciones con los clientes o Customer Relationship Management (CRM), que se considera un elemento estratégico que permite a las empresas mejorar su gestión, sobre la base de una intensa orientación al cliente, al reforzar su imagen de marca y aumentar la cuota de mercado frente a la competencia (Choudhurya y Harrigan, 2014). Esta idea está en sintonía con Zaliznyak (2013:72) cuando afirma que "integrar el pensamiento tradicional del CRM con el CRM social nos permite ofrecer un enfoque nuevo y holístico de estrategia de gestión de relación con el cliente". Todo esto es aún más relevante si consideramos el papel que desarrollan los social media en el turismo desde la perspectiva del cliente. Esto es, el uso de los social media está cambiando profundamente la forma en la que los turistas se informan sobre los servicios que precisan (e.g., vuelos, hoteles, alquiler de coches, etc.), a la vez que producen información de forma colaborativa sobre proveedores turísticos y destinos turísticos (Li y Chang, 2016). Toda esta información puede ser utilizada por las empresas del sector para desarrollar procesos de marketing, que proporcionen nuevos servicios o servicios más ajustados a las necesidades específicas del cliente (Zeng y Gerritsen, 2014).

Por la novedad del SCRM, en la literatura actual existen muy pocos trabajos de investigación que estudien su implementación y los resultados organizativos derivados de su aplicación (Hasani et al., 2017), agravándose este hecho en el ámbito turístico (Diffley et al., 2018). Por todo ello, en el presente trabajo se explora la importancia de los social media y su relevancia en la gestión de las relaciones con los clientes en empresas vinculadas al sector turístico, respondiendo a la necesidad existente en la literatura (Leung et al., 2013). Para alcanzar este objetivo, el trabajo se ha estructurado en cuatro grandes apartados. Así, en el segundo apartado se realiza la revisión de la literatura, en la que se presenta la gestión de la relación con los clientes y la inclusión de los medios sociales en dicha gestión, así como el estado actual de esta cuestión en las empresas turísticas. En el tercer apartado se expone la metodología a seguir. A continuación, en el cuarto apartado se analizan los resultados del estudio, para concluir, 
finalmente, en el apartado cinco en el que se comentan las conclusiones e implicaciones de la investigación.

\section{REVISIÓN DE LA LITERATURA}

\subsection{Customer Relationship Management (CRM)}

Gran parte del éxito de las empresas de servicios se basa en el éxito de la interacción entre el empleado y el cliente (Berry y Parasuraman, 1991). Esta interacción hace posible la creación de una relación entre ambos que se sustenta en algo más que en un conjunto de transacciones y se convierte en un vínculo duradero (Grönroos, 1995). Entre las empresas que siguen esta orientación relacional prevalece la idea de mantener la fidelidad de sus clientes actuales frente a la captación de nuevos clientes, mediante la oferta de productos y servicios ajustados a las necesidades y expectativas de cada individuo. Por tanto, será indispensable averiguar cuáles son aquellos clientes más rentables para la entidad y así establecer una estrecha relación con ellos con el objeto de generar confianza, conocer mejor sus necesidades y aportar ventajas competitivas que impidan que el cliente se sienta atraído por la competencia. De esta forma, ambas partes colaboran en la búsqueda de un beneficio mutuo (Christopher et al., 1994).

Así pues, con el fin de ofrecer un buen servicio y ser capaz de satisfacer unas necesidades concretas, estas empresas tienen que personalizar su oferta atendiendo a las diferentes características que puedan presentar los distintos clientes. Esta necesidad impulsa a las empresas de servicios a desarrollar la CRM entendida como "[...] una estrategia para la gestión de la creación de valor dual, el uso inteligente de los datos y la tecnología, la adquisición del conocimiento de los clientes y la difusión de ese conocimiento a los grupos de interés adecuados, el desarrollo de relaciones apropiadas a largo plazo con clientes específicos y/o grupos de clientes, y la integración de procesos a través de numerosas áreas de la empresa y a través de las redes de empresas que colaboran para generar valor al cliente" (Boulding et al., 2005:157). La aplicación de un buen programa de CRM, según Chaffey (2003), puede concretarse en tres etapas: (1) el cliente es conseguido a través del uso de una comunicación clara sobre una poderosa proposición de valor; (2) el cliente es retenido por el buen servicio ofrecido; y (3) la relación se mantiene en el tiempo a través de la entrega de productos y/o servicios elaborados para segmentos de clientes claramente definidos.

Para lograr tal objetivo, es imprescindible que las empresas de servicio utilicen una serie de herramientas y técnicas innovadoras que les permitan obtener y procesar de forma más efectiva la información obtenida y cuyo análisis lleve a mejorar la gestión de clientes y a diferenciarse de la competencia (Nguyen et al., 2007). Además, les va a ayudar a crear una interacción más personalizada con el cliente lo que les podría conducir, no sólo a captar nuevos clientes, sino a fidelizarlos a la marca (King y Burgess, 2008). Palazón et al. (2014) formulan la idea de que actualmente no es suficiente con que una empresa genere satisfacción hacia una marca, sino que es necesario que el cliente se sienta parte de ella de una manera más emocional y afectiva. Las marcas que gozan de valor emocional por parte de los consumidores se posicionan en el mercado mejor que su competencia. Esto es, aquellas empresas que consigan generar ese sentimiento cercano y positivo hacia sus clientes contarán con una ventaja competitiva que les permitirá elevar los precios de sus servicios. Estos clientes fieles preferirán acudir a estas empresas ya que les aportan mayor satisfacción y aprecian más que aquellas de la competencia. Así pues, la incorporación de un programa de CRM va 
a permitir identificar y conocer mejor a los clientes actuales, al contar con información más detallada y centralizada sobre ellos, que les lleva a personalizar los servicios de manera más precisa, evitando errores y la obtención de datos incoherentes (Winer, 2001; Rodriguez et al., 2012).

Una de las ventajas que obtienen las empresas que tienen identificado su grupo de clientes fieles es que, a la hora de realizar campañas, éstas se centran más en orientar las actividades de promoción hacia ese grupo específico de clientes que a captar nuevos clientes. Al reservar esos recursos, las organizaciones pueden proporcionar mayor calidad, una atención más personalizada, mejorando los sistemas de información y comunicación, ofreciendo programas de fidelización, etc. (Nguyen et al., 2007; Faase et al., 2011). Asimismo, las empresas que cuentan con clientes leales disponen de vendedores de prestigio, ya que a través del boca-oreja, éstos son capaces de difundir el mensaje contando sus propias experiencias sobre la empresa y los servicios (HennigThurau et al., 2002; Yen y Gwinner, 2003). Además, si un cliente está satisfecho con el servicio recibido, éste no tendrá problemas en pagar un precio mayor que el de competencia. Del mismo modo, al tener noción de los gustos de cada individuo, la empresa hace posible el uso de la venta cruzada, que permite vender más artículos o servicios a un cliente en el mismo acto de venta. Otra de las ventajas que se debe resaltar, es que un cliente fiel suele dirigirse a ese comercio con más frecuencia, ya que anteriormente ha contado con una experiencia satisfactoria (Chang et al., 2002). Sin embargo, es preciso señalar que llevar a cabo un proyecto de CRM implica una mayor atención al cliente, por lo que los costes internos se ven afectados, ya que el coste de esfuerzo y tiempo que se debe dedicar a cada cliente se eleva. A pesar de ello, la literatura indica que el resultado es muy favorable, ya que las empresas cuentan con clientes más fieles y conocedores de la empresa, los cuales están más familiarizados con los servicios que se prestan y las dudas sobre la empresa y el producto son menores (Cambra-Fierro et al., 2014). Al mismo tiempo, los problemas se resuelven más rápidamente porque se tiene constancia de mucha información detallada sobre el cliente y sobre qué productos tiene contratados, la cual se encuentra volcada en una base de datos (Hackney, 2000; Wood, 2000). Por tanto, el CRM apoya al marketing, las ventas y cuida al cliente de una forma eficaz y eficiente (Kim et al., 2003), lo que contribuye a mejorar los resultados empresariales (Chang et al., 2002; Boulding et al., 2005; Sigala, 2005).

\subsection{Social Customer Relationship Management (SCRM)}

A medida que la tecnología avanza, la sociedad ha empezado a comunicarse y a compartir opiniones a través de los medios sociales, lo que ha provocado el interés de las empresas al reconocerlos como un método efectivo para mantener relaciones duraderas con los clientes (Trainor, 2012). Tal y como señalan Choudhury y Harrigan (2014:150), "hoy en día, con la llegada e inclusión de la naturaleza de una Web 2.0 avanzada, utilizada en las tecnologías de los medios sociales, la CRM tiene otra oportunidad de crecer y prosperar". La Web 2.0 permite a los consumidores modificar la forma en la que buscan, leen, comparten, comunican y crean información, transformando las formas en las que se crea valor para el cliente (Sigala, 2011). Es por ello que surge un nuevo concepto: SCRM o CRM 2.0 (llamado así por el uso de la Web 2.0). El SCRM es una actualización del concepto tradicional de CRM, en el que se torna insuficiente la comunicación individualizada para un determinado cliente, debiendo producirse entre cliente y cliente y dentro de las redes de empresas y clientes, al objeto de poder crear valor (Sigala, 2011). 
Aun siendo un concepto muy novedoso, es objeto de estudio actual por numerosos autores de la literatura de marketing, tal y como se presenta en la Tabla 1.

\section{Tabla 1. Definición del concepto de SCRM}

Autor y fecha

Mohan et al.

(2008:241)

(2010:19)

Faase, Helms y

Spruit (2010:7)

Greenberg

(2010:414)

Social CRM son aplicaciones independientes fáciles de usar que pueden aprovecharse en los procesos estructurados de CRM existentes para ayudar a los usuarios finales a explotar mejor las redes sociales, las fuentes de datos, las noticias internas y externas, y el contenido de ventas y marketing existente.

Social CRM es una filosofía y una estrategia de negocio, soportada por un sistema y una tecnología, diseñada para implicar al cliente en una interacción colaborativa que proporcione valor mutualmente beneficioso, en un entorno de negocio de confianza y transparencia.

No se trata de sustituir al CRM clásico, sino de enfatizar en la «R» de Relación y abrirla a las redes sociales; de este modo humanizamos la empresa, y los clientes ya no se relacionan con una marca abstracta y distante, sino con gente que representa a una marca con la que se sienten identificados.

Social CRM trata de la creación de una interacción de doble sentido entre el cliente y la empresa. Es una estrategia de CRM que usa los servicios de la Web 2.0 para animar la vinculación e implicación activa del cliente.

Social CRM es una estrategia de negocio que involucra a las áreas de marketing, ventas y procesos de servicio al cliente para incluir la participación activa de los clientes o visitantes en un canal de Internet (web o móvil) con el objetivo de fomentar la participación en el proceso de negocio.

El CRM 2.0 es como una solución de marketing basada en el diálogo, con el apoyo de herramientas de sistemas de información, donde no sólo se quiere vender, sino conocer las reacciones de los clientes, el por qué no están satisfechos, por qué les gustan nuestros productos, cuáles son sus problemas y cómo podríamos satisfacerlos de nuevo.

Woodcock, Green, y Starkey

SCRM es una estrategia de negocios que implica al cliente a través de los (2011:52) social media con el objetivo de construir confianza y lealtad hacia la marca.

Trainor (2012:319)

Yoon y Sims (2013)
SCRM consiste en la integración de las actividades tradicionales a las que se enfrenta el cliente, incluyendo procesos, sistemas y tecnologías, con aplicaciones de social media emergentes, con el fin de implicar a los clientes en conversaciones colaborativas y mejorar las relaciones con él.

Se entiende por Social CRM la práctica comercial de promover actividades voluntarias de participación del cliente, impulsadas por una combinación de redes sociales, en el proceso de creación de valor para construir relaciones 
co-beneficiosas a largo plazo con los clientes objetivo bajo el paraguas de la gestión de las relaciones con los clientes.

Harrigan y Miles Social CRM es la incorporación de las tecnologías cotidianas de redes $(2014: 100)$ sociales en el arsenal del CRM.

Fuente: Elaboración propia

De acuerdo con estas definiciones, el diálogo, la participación y la colaboración con el cliente se erigen en ejes centrales del CRM, facilitados por el uso de los medios sociales, lo que va a posibilitar el desarrollo del SCRM. Sin embargo, el SCRM no debe ser visto únicamente como la aplicación de las tecnologías de la información al CRM, sino como una combinación de hardware, software, capital humano, procesos, aplicaciones y compromiso directivo cuyo objetivo es atraer y retener a los clientes valiosos económicamente y mejorar la efectividad del marketing para crear y entregar el máximo valor a los clientes (Fjermestad y Romano, 2003; Scullin et al., 2004; Faase et al., 2011). Así, gracias a los medios sociales, es posible establecer relaciones más intensas con los consumidores, donde los clientes han dejado de ser meros lectores y pasan a ser plenamente activos, esto es, a participar mostrando sus opiniones y experiencias tanto positivas como negativas, información, fotos, videos, etc. (Caro Herrero et al., 2015), permitiendo a las empresas conocer qué es lo que gusta o no, así como conocer cuáles son sus fallos para poder repararlos (Sigala, 2016). El cliente actual es un cliente social, por lo que el SCRM no es sólo una herramienta que permite vender más, sino que posibilita conocer exactamente lo que se demanda (Bagó y Vörös, 2011). Esto es, el SCRM facilita el diálogo abierto, lo que crea una interacción dinámica entre ambas partes y da a los consumidores la propiedad sobre la conversación con la empresa. Esto permite que se genere la oportunidad de co-crear valor para el cliente, resultando en un enfoque realmente centrado en el cliente (Chen y Vargo, 2014). De esta forma, se puede evitar el lanzamiento de un producto al mercado con la incertidumbre de si el cliente lo aceptará y comprará, ya que teniendo constancia de cuáles son sus expectativas, los resultados serán mucho más exactos (Faase et al., 2011). Además, la literatura sugiere que el uso de los social media en el CRM podría influir en el resultado organizativo, motivado por la mayor implicación del cliente, el aumento de las interacciones y el incremento de información compartida a través de las redes sociales (Agnihotri et al., 2012; Brodie et al., 2011; Hennig-Thurau et al., 2010; van Doorn et al., 2010; Choudhury y Harrigan, 2014).

\subsection{SCRM en las empresas turísticas}

El CRM es uno de los enfoques de dirección que más rápidamente está siendo desarrollado e implantado por numerosas organizaciones (Trainor et al., 2014), predominantemente por empresas turísticas y de alojamiento (Sigala, 2003). Mantener relaciones con los clientes y empleados es una actividad fundamental para las empresas de servicios y especialmente las relacionadas con el turismo tales como hoteles, agencias de viajes, restaurantes, casinos o líneas aéreas (Maggon y Chaudhry, 2015). Para lograrlo, y dado el impacto potencial de los social media en el turismo, es necesario estudiar en profundidad su papel, al objeto de mejorar las prácticas de marketing en el sector turístico (Rosman y Stuhura, 2013). 
El interés de los investigadores por el estudio del SCRM en la industria turística es reciente y va en aumento (e.g., Sigala 2011; Almunawar et al., 2012; Bygstad y Presthus, 2012; Rosman y Stuhura, 2013; Liau y Tan, 2014; Dieck et al., 2017; Lo et al., 2017; Diffley et al., 2018). Así, mientras unos autores estudian el uso de los social media en el CRM en las empresas turísticas en general (e.g. Sigala, 2011;2016), otros lo analizan en el contexto hotelero (e.g. Rosman y Stuhura, 2013; Lo et al., 2017; Dieck et al., 2017; Diffley et al., 2018), en agencias de viajes (e.g. Almunawar et al., 2012) o en líneas aéreas (e.g. Bygstad y Presthus, 2012). Sin embargo, es sorprendente que el número de investigaciones realizadas sobre este tópico en España sea aún escaso (Garrido-Moreno y Padilla-Meléndez, 2012), sobre todo si se tiene en cuenta el impacto económico de esta industria en el país.

España es un país reconocido por su actividad turística, al que acudieron más de 75 millones de viajeros en el último año, atraídos por sus playas, el abundante patrimonio artístico y la variedad de oferta gastronómica. Según el ranking mundial de competitividad turística elaborado por el World Economic Forum en 2017, España se encuentra situada en primer lugar gracias a sus recursos naturales y culturales, las infraestructuras turísticas, la conectividad del transporte aéreo y el fuerte apoyo político a la industria turística. El desafío al que se enfrenta hoy en día el turismo en España consiste en seguir buscando nuevas formas de mejorar a través de acciones que modernicen la industria, dado que es un sector que se encuentra en madurez. Entre estos aspectos de modernización se encuentra la mejor preparación de la industria para el uso de las TICs (uso de internet para las transacciones B2C, y de las TICs para las transacciones B2B, entre otras), al ocupar actualmente España el puesto número 29 de los 136 países que componen el estudio. Este esfuerzo ha comenzado y muchas empresas del sector turístico español ya están adecuándose a los hábitos de consumo digitales demandados por los turistas. Aunque el reto es, si cabe, aún mayor, al tener que incluir la utilización de los medios sociales, lo que está revolucionando las estrategias de marketing desarrolladas por estas empresas (Hosteltur, 2017). Según Hosteltur (2017), tener la capacidad de invertir el tiempo y el esfuerzo necesario para obtener un retorno real de las acciones de social media será determinante para optimizar los presupuestos y obtener el máximo rendimiento.

En cuanto a Canarias, son factores como su clima y su biodiversidad lo que hacen que el Archipiélago mantenga su situación de privilegio como destino turístico dentro de España y en el mercado internacional. El turismo se considera el motor del desarrollo económico de Canarias y el sector con más capacidad de generar empleo. De hecho, el peso de la actividad turística en el conjunto de la economía canaria se cifró en el 31,9\% del PIB en 2015 (Impactur, 2016). Este importante dato viene apoyado por los 15 millones de turistas que visitaron las Islas Canarias en 2016, lo que significa que el Archipiélago absorbe el $20 \%$ de los turistas que visitan España, según cifras del ISTAC. De forma específica, y según esta misma fuente, la isla de Gran Canaria acogió a un total de 4.334.679 turistas en 2016, suponiendo casi un 30\% de los visitantes del Archipiélago. Desde el punto de vista del índice de penetración de las TIC en el sector turístico canario, César Miralles, director de Red.es, comenta que entre las pymes y grandes empresas la conexión a Internet la tiene el 98\% de las mismas en España, mientras que en Canarias es del 97,3\%. Sin embargo, entre las microempresas - hasta 9 empleados - disponen de conexión a la red un $65,7 \%$ a nivel nacional y en Canarias se reduce a un $58,1 \%$. Asimismo, opina que esta situación sigue siendo un reto y una prioridad en la Agenda Digital del Ministerio, no detectando grandes diferencias entre el sector turístico canario y el del resto de España porque es un sector innovador y potente. 
Ante estos datos, son numerosas las empresas del sector turístico canario que comienzan a hacer gran hincapié en las redes sociales para darse a conocer y fidelizar a aquellos turistas que ya han visitado la isla anteriormente. Estas empresas son conscientes de la necesidad de estar presente en los medios sociales, tener la información actualizada e interactuar con los usuarios, ya que uno de sus usos más frecuentes consiste en consultar comentarios y referencias de otros usuarios. De hecho, Promotur Turismo Canarias publicaba recientemente que una encuesta realizada en Reino Unido situaba a las Islas Canarias como el quinto destino más comentado a nivel internacional en Facebook y Twitter por los usuarios de aquel país, solo por detrás de Nueva York, París, España y Francia. El estudio destacaba que cuatro de cada diez adultos del Reino Unido admiten ser "turistas sociales" que consultan en las redes sociales información de un promedio de 14 destinos turísticos todos los años. Además, refleja que tres de cada diez personas afirman que las redes sociales son su mayor influencia a la hora de elegir su próximo destino vacacional.

En esta misma idea inciden Hanna et al. (2011) cuando señalan que, a la hora de comprar billetes de avión, alquilar un coche o una habitación de hotel, muchos usuarios prefieren informarse a través de las opiniones de otros que hayan adquirido esos servicios previamente o de otra información que no especifica la empresa en su página web. Estos comentarios, tanto si son positivos como negativos, influirán en la decisión de compra de los clientes. Con la aparición y uso de los medios sociales, las empresas adquieren conocimiento de sus clientes, lo que hace que se encuentren en una situación de privilegio frente a sus competidores (Liau y Tan, 2014). Sin embargo, hoy en día, esa oportunidad ha pasado a ser una necesidad. Las empresas turísticas son conscientes de que además de encontrarse en los medios sociales es preciso gestionarlos de forma efectiva, ya que si este "boca/oreja 2.0" no se sabe tratar adecuadamente puede llegar a convertirse en una desventaja que no solo reducirá las ventas de la empresa, sino que puede llegar a empeorar la imagen corporativa (Munnukka y Järvi, 2014). Esto es, las empresas deben implementar de forma estratégica el SCRM, de tal forma que el uso de la Web 2.0 sirva para fomentar la colaboración entre los clientes y, entre éstos y la empresa con el fin de mejorar y compartir el conocimiento (Faase et al., 2011). Es por ello, que en un futuro próximo las empresas vinculadas con el turismo deberán ir integrando más intensamente los social media en las plataformas de gestión de clientes, lo que dependerá principalmente del nivel de madurez de los social media de la empresa. Así, de acuerdo con Thryambakam y Bethapudi (2013), aquellas empresas que se encuentren en un estadio inicial necesitarán un plan de social media más amplio e integral. Por tanto, es necesaria la implicación y el compromiso de la dirección en el desarrollo del SCRM, sobre todo al tomar consciencia del papel que juega la tecnología en la generación de capacidades que mejoran los resultados organizativos (Trainor et al., 2014).

Ante este escenario, un SCRM bien gestionado pasa ser una herramienta clave en la estrategia a seguir por las empresas del sector, ya que permite mejorar la lealtad de los clientes, además de lograr la diferenciación frente a la competencia. De este modo, la recopilación y almacenamiento de información de interés sobre clientes y el acceso a ésta por toda la organización, permite a los empleados usarla de forma efectiva para prestar un mejor servicio personalizado y convertirlo en una experiencia superior para el consumidor (Frow y Payne, 2009; Chathoth et al., 2013), que derive en mejores resultados para las empresas del sector (Diffley et al., 2018). 


\section{METODOLOGÍA}

El objetivo principal del presente trabajo consiste en explorar la importancia de los social media y su relevancia en la gestión de las relaciones con los clientes en empresas vinculadas al sector turístico. Para dar respuesta a este objetivo se va a llevar a cabo una investigación de carácter cualitativo con una doble finalidad: (1) va a permitir explorar y profundizar en un área aún incipiente y sobre la que existe un escaso conocimiento previo; (2) su utilización permitiría explicar cómo el SCRM puede incidir en la fidelización de los clientes en el sector turístico, sobre la base del conocimiento de las actitudes y predisposición de sus directivos, las necesidades de las empresas que conforman esta industria, sus motivaciones, etc.

Desde el punto de vista metodológico, la presente investigación ha consistido en la realización de tres extensas entrevistas en profundidad a directivos de importantes empresas relacionados con el sector turístico en Gran Canaria (Cadena de agencias de viajes, Cadena hotelera y Línea aérea). Estas entrevistas van a permitir contextualizar otros datos, como los de los resultados, ofreciendo una fotografía más completa de lo que ocurre y por qué, además de proporcionar una información más rica y detallada del tema a explorar (Boyce y Neale, 2006). Las preguntas de la entrevista se elaboraron a partir de la literatura del CRM y SCRM y fueron diseñadas de forma amplia debido a la naturaleza exploratoria propia de este estudio. Inicialmente, se pidió a los encuestados que describieran algunos datos generales sobre la posición que ocupan en la empresa, cuál es la actividad principal de la misma, qué tipo de clientes son a los que se dirigen y la facturación aproximada alcanzada el año anterior. Posteriormente, se realizaron preguntas consistentes en conocer la importancia que tiene para cada una de estas importantes empresas llevar una buena gestión de relaciones con los clientes a través de los medios sociales, y su opinión sobre el impacto que tiene el SCRM en el sector turístico actualmente. Además, se les preguntó por el grado de implantación de esta estrategia de gestión de clientes (por ejemplo, si existía un departamento específico para ello, el tipo de actividades que realizaban, el nivel de tecnología que manejaban, el grado de desarrollo de los social media, etc.). Otra de las cuestiones tenía como finalidad conocer su apreciación sobre los beneficios de su implantación. Finalmente, se les pidió su opinión personal sobre la perspectiva futura que tiene SCRM tanto para el sector turístico en general, como para la empresa que dirigen en particular.

\section{RESULTADOS}

Con el fin de exponer de forma clara y comprensible la cantidad de información obtenida tras la realización de las entrevistas en profundidad, se van a exponer los resultados del estudio destacando las similitudes y diferencias que existen entre las diferentes opiniones vertidas por los directivos entrevistados, agrupadas en 4 apartados.

\section{- Recursos para la fidelización del cliente en las empresas turísticas.}

En este apartado se expondrán cuáles son los recursos con los que cuentan las empresas del sector turístico entrevistadas. En general, las tres empresas utilizan algunas técnicas de fidelización dirigidas a sus clientes. Sin embargo, debido al tamaño de cada una de las empresas, los recursos con los que cuentan y las estrategias que llevan a cabo, las actividades que realizan para fidelizar al cliente son diferentes. Por una parte, en el caso de la aerolínea, el responsable de la fidelización de la empresa explicó que ésta cuenta con un departamento de gestión de clientes, cuyo numeroso 
personal se encarga de todos los trámites relacionados con la fidelización y atención de clientes. Por otro lado, en el caso de la cadena de agencias de viajes, el directivo comentó:

"Hoy por hoy no tenemos un plan de fidelización de clientes definido, no tenemos un departamento, ni tan siquiera recursos que estén destinados específicamente a ese aspecto. Sí que lógicamente actuamos en muchos casos para fidelizar a nuestros clientes, pero basándonos en la experiencia, más que por una estrategia creada y diseñada"

En el caso de la cadena hotelera, actualmente no tienen un departamento específico que se encargue de la fidelización de clientes, ya que esta actividad está gestionada por el departamento comercial. Sin embargo, el entrevistado explicó que sus acciones de fidelización pasan por explotar de forma adecuada su base de datos de clientes. En este caso, este directivo comentó:

"Tenemos una base de datos a través de la que el hotel sabe cuándo el cliente ha repetido un determinado número de veces y, según esto, se le aplica un protocolo; se le ofrece bebidas o algún tipo de amenitie especial en la habitación, se le da la bienvenida por su nombre... como con un trato de privilegio"

Los tres entrevistados consideran la fidelización de sus clientes fundamental para la empresa, por lo que este hecho representa en la consecución de una ventaja competitiva sostenible y el éxito empresarial a largo plazo (Kandampully y Suhartanto, 2000). Así, en el caso de la aerolínea, dado que poseen un departamento de fidelización y una estrategia de gestión de clientes claramente definida, es posible ver los resultados y beneficios que supone llevar un control sobre las actividades que dirigen a sus clientes, ya que facilita la planificación de la comunicación y la oferta de productos y servicios, entre otros, atendiendo a los diferentes segmentos de sus clientes actuales. Además, según este directivo, mantener un programa de fidelización también hace más fácil la realización de las distintas campañas de captación de nuevos clientes que se ejecutan periódicamente. Esto es así, ya que cuentan con información sobre los clientes actuales, lo que les permite conocer mejor cómo deben dirigirse a otros nuevos para captarlos más fácilmente. La importancia de incrementar la fidelización del cliente trae consigo una mejora en los resultados económicos de la empresa motivada, entre otros, por un mayor éxito en el incremento de la venta cruzada a los clientes fieles, un boca-oreja más positivo, costes de marketing más reducidos al disminuir sus esfuerzos por atraer nuevos clientes, y el fortalecimiento de la posición de la empresa en el mercado (Griffin, 2000).

- Evaluación de la gestión de las relaciones con los clientes en las empresas turísticas.

En relación a esta cuestión, los tres directivos coincidieron en afirmar que sus empresas seguían un programa de gestión de CRM, sin embargo, éste se desarrolla y ejecuta de forma diferente en cada caso. Así, por ejemplo, la aerolínea cuenta con una 
aplicación propia de CRM desde hace más de 10 años y un importante sistema de almacenamiento de datos donde recopilan los datos históricos recogidos de la web, las facturaciones, los check in, etc. Por su parte, la agencia de viajes utiliza su propio sistema de facturación que le permite recoger datos sobre el cliente y posteriormente medir y conseguir información valiosa con el fin de segmentar y destinar sus esfuerzos a los distintos tipos de clientes. De esta forma, el sistema de facturación de esta empresa, creado desde la apertura de la misma, se ha ido perfeccionando con el tiempo y es considerada como una herramienta potente de CRM. Sin embargo, el directivo de esta empresa afirma que

"[...] aunque tenemos el programa de CRM y el software capacitado para ello, hasta ahora no lo hemos desarrollado en gran medida, aunque es uno de sus objetivos a alcanzar en el futuro".

Asimismo, la dirección es consciente de las ventajas que implica desarrollar un programa de CRM, ya que, aunque no aún no han generado beneficios fruto de su puesta en marcha, reconocen su potencial para generar conocimiento acerca de sus clientes y adelantarse a sus expectativas y necesidades, en la misma línea que apuntan Rodriguez et al. (2012). Respecto a la cadena hotelera, la empresa utiliza su sistema de reservas como CRM para adquirir información sobre sus clientes, aunque la mayor parte de esa información la recoge de los turoperadores con los que trabaja, ya que son éstos quienes tienen contacto directo con el cliente. Además, este directivo señala que la empresa utiliza una herramienta que resume y analiza el contenido online generado por los usuarios a través de las agencias de viajes y redes sociales. De esta forma, la empresa está capacitada para escuchar y responder activamente a los comentarios de los clientes, mejorando su experiencia e incrementando los resultados organizativos (Trainor et al., 2014).

En general, todas las opiniones coinciden en resaltar la importancia de una gestión efectiva de las relaciones con los clientes al objeto de aumentar la eficiencia en los servicios prestados, coincidiendo con lo señalado por Teo et al. (2006). Así, por ejemplo, un directivo comentó que, desde la implantación del programa de gestión de CRM en la empresa, la comunicación y las campañas que desarrollan son más eficientes, en cuanto no saturan al cliente con información inútil y permiten que se reduzcan los costes (Griffin, 2000).

- El uso de los social media en las empresas turísticas.

Actualmente, las empresas analizadas cuentan con perfiles en distintos medios sociales, siendo los más usados Facebook, Twitter, LinkedIn y YouTube, mientras que Instagram, Pinterest y Google+ son utilizados solo por alguna de ellas. Los directivos entrevistados coinciden en la importancia de estar presentes en las redes sociales, señalando su participación desde hace años en Facebook y Twitter, aunque sin contar con una estrategia específica que le permitiera gestionar estos medios. Sin embargo, en todos los casos los directivos son conscientes de que han de profundizar más en este tema, debido a la importancia estratégica que adquieren los social media como herramienta para escuchar e interactuar con el cliente, que les va a permitir mejorar la comprensión de los mercados, clientes, competidores y empleados (DiStaso et al., 2011), así como en la competitividad turística (Leung et al., 2013). 
Así, en el caso de la cadena de agencia de viajes, inicialmente contaba con una presencia testimonial en los medios sociales, sin planificación alguna. Sin embargo, hoy en día la empresa ha conformado una mesa 2.0, compuesta por el director comercial, responsable de marketing y director de la agencia, en la que se definen los objetivos y estrategias a seguir en los medios sociales. Esta empresa cuenta con una estrategia de contenidos definida en sus redes sociales, en la que muestran contenidos que interesan al cliente (por ejemplo, la creación de publirreportajes de aquellos destinos que publicitaba la empresa). De esta forma, la empresa es capaz de llegar al cliente de una manera mucho más personal y de ofrecer información valorada por el cliente. En cuanto al caso de la aerolínea, si bien no contaban inicialmente con una planificación de sus social media y principalmente informaban sobre productos y ofertas sin que existiera una relación empresa-cliente, hoy en día, la gestión de los social media de la empresa es un reto para el que han destinado recursos tales como la contratación de un community manager. Finalmente, la cadena hotelera cuenta con un departamento de comunicaciones en el que se ha invertido en personal cualificado para gestionar toda la información vinculada a las redes sociales de la empresa. Esta empresa cuenta con un perfil general en Facebook y Twitter en el que se ofrece información sobre la cadena y otros perfiles independientes para cada uno de los hoteles que la componen. De esta forma, se logra dar información más específica que activa el interés de los clientes reales y potenciales (eventos y actividades semanales del hotel, comentarios y fotos de las diferentes acciones realizadas, campañas que se están lanzando, ofertas puntuales, etc.). Todas las acciones de comunicación que la empresa expone en las redes sociales deben ir alineadas con las estrategias de la empresa (Valos et al., 2016).

Sin embargo, los directivos deben tomar consciencia de que, además de conocer qué herramientas deben usar y cómo usarlas apropiadamente, han de interesarse en cómo medir su efectividad (DiStaso et al., 2011). Es por tal motivo, que las tres empresas entrevistadas realizan análisis periódicos sobre el número de visitas, fans que se incorporan a la empresa, retweets, etc., reconociendo el reto que deben afrontar. Para ello, las empresas opinan que es necesario comenzar por realizar reuniones periódicas al objeto de conocer los avances en social media, así como desarrollar análisis de la efectividad de las acciones realizadas, entre otros. Todos coinciden en el estadio inicial en el que se encuentran en relación al conocimiento, implementación y medición de los social media, aunque consideran que es uno de sus objetivos a desarrollar a corto plazo. Así, por ejemplo, uno de los propósitos de la cadena de agencias de viajes es crear un cuadro de mandos que permita conocer los indicadores que señalan el éxito o fracaso de las diferentes acciones que realice la empresa, ya que, según este directivo,

"[...] todo lo que siempre se dice, todo lo que se hace en redes sociales u online, en general, debe ser susceptible de ser medido, porque si no, no tiene sentido que se haga".

\section{- Valoración del SCRM en el sector turístico.}

En relación a la relevancia del SCRM en el sector turístico, todos los entrevistados coinciden en afirmar que existe una necesidad imperiosa de gestionar de forma efectiva los medios sociales en la industria. La literatura apoya esta premisa sobre la base de numerosas investigaciones que han estudiado la aplicación de los social media en el sector turístico (e.g., Chan y Denizci Guillet, 2011; Li y Wang, 2011; Noone et al., 2011; Xiang y Gretzel, 2010; Leung et al., 2013). Los directivos aluden a la necesidad 
de su desarrollo motivado, principalmente, por la relevancia de este sector en la economía, la fuerte penetración de los medios sociales y el gran número de usuarios que los utilizan como herramienta de información decisiva para la compra de productos y/o servicios. Además, coinciden en que los medios sociales actúan como impulsores de relaciones bidireccionales con los clientes, imprimiéndoles dinamismo, y capacitando a las empresas para conocer mejor a sus clientes y dar respuesta a sus problemas, al tiempo que crean y mantienen vínculos más estrechos con los usuarios, tal y como señalan Tsimonis y Dimitriadis (2014). De hecho, Salkhordeh (2009) sugiere que los social media son una herramienta valiosa para recopilar información sobre la experiencia del consumidor con el servicio, lo que podría servir para atraer a nuevos clientes y mantener a los actuales a través de la creación de relaciones fuertes y efectivas que fortalezcan de lealtad a la marca. Además, este autor indica que la gestión estratégica de la información en las redes sociales es clave para que la empresa determine si las adopta para el negocio, además de serlo para los ingresos.

Por otra parte, los entrevistados con conscientes de que se encuentran en las primeras etapas de la implementación del SCRM en sus empresas, debiendo desarrollar esta estrategia en el futuro. Los directivos entrevistados insistieron en que, aunque se cuente con perfiles en redes sociales, es absolutamente necesario que exista una planificación previa y una gestión adecuada, además de su integración en la estrategia de CRM. En caso contrario, la iniciativa tendría escaso valor. Por ello, el representante de la aerolínea explicó que:

“[...] Las redes sociales constituye un canal más, no puede ser en sí misma la estrategia. Detrás debe existir una estrategia elaborada".

En este sentido, el entrevistado de la cadena de agencias de viajes comparte esta visión, por lo que comenta:

“[...] tenemos que focalizar muchos esfuerzos en la estrategia digital. Debemos utilizar un CRM profesional para conocer al cliente, dinamizar las relaciones y crear la publicidad del siglo XXI."

Además, expresaron la necesidad de combinar distintos canales con el fin de alcanzar diferentes objetivos estratégicos, lo que coincide con lo apuntado por Valos et al. (2016). Así pues, según apuntaron los directivos, carece de sentido desarrollar una estrategia en redes sociales sin contar con una página web que permita ofrecer productos y servicios, de tal forma que se conviertan en fuente de nuevos contactos (Teo y Alt, 2012). Sin embargo, el objetivo del SCRM no consiste únicamente en contactar con los clientes a través de ofertas en la página web o en las redes sociales, sino en construir relaciones fuertes a través de la entrega de una experiencia positiva con la marca, los productos y servicios de la empresa (Van Looy, 2016). Finalmente, Los directivos señalaron que uno de sus objetivos prioritarios en un futuro inmediato va a consistir en profundizar en el análisis de los medios sociales, lo que hasta ahora ha sido insuficiente, añadiendo que confían en que a través del SCRM se podrán mejorar las relaciones con los clientes, lanzar campañas más efectivas, mejorar productos, realizar programas de fidelización acorde a cada tipo de cliente, etc. 


\section{CONCLUSIONES E IMPLICACIONES}

El objetivo de este trabajo consistía en explorar la relevancia del SCRM en la gestión actual de las relaciones con los clientes en empresas vinculadas al sector turístico. Los resultados del estudio ponen de manifiesto, entre otras, la importancia que tiene el SCRM para las empresas de este sector, de acuerdo con las valoraciones expuestas por los directivos entrevistados. Sin embargo, todavía se trata de un término muy novedoso y con escasa aplicación en el sector turístico, lo que coincide con lo señalado por Harrigan et al. (2017), máxime cuando las empresas se encuentran en las etapas iniciales de su implementación y están comenzando a realizar la gestión efectiva de los medios sociales en los que están presentes.

Es en este momento actual en el que estas empresas están valorando la importancia de crear y desarrollar una estrategia efectiva a la hora de estar presentes en los distintos medios sociales para fidelizar al cliente. En los tres casos investigados, hasta hace un año aproximadamente, su presencia online consistía básicamente en un escaparate en los medios sociales para su empresa, sin que existiera ningún tipo de interacción con el cliente. Hoy en día, este proceso sigue aún en estado embrionario, al continuar ocupándose únicamente de analizar los indicadores que les permiten conocer su actividad en los social media en los que operan, sin profundizar en aquellos análisis que certifiquen el avance en su relación con los clientes, la efectividad de las campañas que realizan, la mejora de la imagen de marca, etc. Sin embargo, estas empresas son conscientes de la importancia que tiene mantener relaciones bilaterales y constantes con los clientes por los numerosos beneficios que se pueden obtener.

Es por ello que, a pesar de que las empresas entrevistadas tienen características diferentes, en relación a los servicios que ofrecen, así como en el tamaño y el número de trabajadores en plantilla, todas ellas comparten la necesidad de crear un equipo de trabajo formado por directivos y personal especializado, que se encargue de establecer estrategias y objetivos comerciales y de fidelización para llevarlas a cabo a través de los medios sociales. Estas empresas son conscientes de la relevancia que estos medios tienen para los clientes actuales y potenciales, y del importante aumento de su uso a la hora de adquirir un servicio turístico, de acuerdo con lo que señala Leung et al. (2013). Así, por ejemplo, el $62 \%$ de los usuarios consideran que las valoraciones y comentarios en redes sociales influyen en sus decisiones de compra. Sin embargo, hasta la fecha, estas empresas han concebido el concepto de SCRM desde un enfoque únicamente tecnológico y no desde un enfoque estratégico con el que lograr la fidelización de sus clientes (Cambra-Fierro et al., 2014). Tal y como señalan Rapp y Ogilvie (2016), sin una implementación estratégica adecuada, las empresas son propensas a fallar en sus intervenciones en los medios sociales, corriendo el riesgo de dañar la relación con el cliente más rápidamente de lo que consiguen mejorarla. Poseer simplemente un perfil en redes sociales es insuficiente. La clave está en entender y gestionar intensamente la información obtenida de la colaboración con el cliente, al objeto de conseguir una ventaja sobre los competidores (Diffley et al., 2018). Desarrollar una visión estratégica del SCRM, respaldada por la inversión tecnológica de redes sociales, capacitaría a la empresa para aprender de sus clientes fruto de la mayor interacción con la empresa y con otros clientes; para escuchar lo que opinan de la marca, así como de los productos o servicios que ofrecen, y para responderles de forma inmediata (Faase et al., 2011; Trainor et al., 2014). 
Sin embargo, esta visión estratégica no corresponde únicamente al departamento de comercial y de marketing. La implantación del SCRM requiere de una visión estratégica que debe impregnar toda la organización, así como a sus procesos y empleados, en la que la orientación al cliente se convierta en el aspecto clave para lograrlo (Teo et al., 2006). Este enfoque integrado facilita la obtención, interpretación y gestión de la información de los medios sociales y canales tradicionales, logrando un trato integral del cliente y un mayor nivel de intercambio. Así, a través de esta información, las empresas "[...] mejoran la experiencia del cliente, resultando en el desarrollo de nuevos modelos innovadores para la implicación del cliente" (Baird y Parasnis, 2011:32). Por tal motivo, se hace necesario realizar algunas recomendaciones a las empresas vinculadas con el turismo en Gran Canaria, de tal forma que puedan ser consideradas a la hora de implementar el SCRM de forma efectiva.

Así pues, en primer lugar, las empresas del sector turístico han de ser conscientes de que el SCRM no es sólo un conjunto de tecnologías, sino una estrategia encaminada a comprender mejor las necesidades de los clientes y crear fuertes vínculos con ellos, para lo que habrán de hacer un esfuerzo intenso y continuado en la mejora de tales relaciones (Faase et al., 2011; Nguyen et al., 2007), desarrollando prácticas colaborativas con las que se compartan ideas y sean fuente de innovación (Baird y Parasnis, 2011; Diffley et al., 2018).

En segundo lugar, es preciso que estas empresas cuenten con el compromiso firme de la alta dirección, con un presupuesto que apoye la estrategia, de tal forma que se garantice su implementación y continuidad, así como con el fomento de una comunicación fluida entre los miembros de la organización (King y Burgess, 2008). Este apoyo directivo sentaría las bases para el desarrollo del SCRM, al tiempo que garantizaría cierta inversión en tecnología, el rediseño de procesos y la contratación o adjudicación de personal específico para ello, con el fin último de lograr una mejor comprensión de las necesidades de los clientes (Garrido-Moreno y Padilla-Meléndez, 2012).

En tercer lugar, sería conveniente que el personal fuera motivado y recompensado por su implicación en actividades propias del CRM y el despliegue de comportamientos orientados al cliente, de acuerdo con lo señalado por Reinartz et al. (2004), Özgener y Iraz (2006) y King y Burgess (2008). Para conseguir tal fin, es necesario que los empleados que participen en este programa sean formados previamente, al objeto de desarrollar o mejorar sus habilidades y competencias tecnológicas y, así, conseguir mejorar la explotación de la Web 2.0 de las empresas de este sector, coincidiendo con lo que apunta Sigala (2011), Baird y Parisnis (2011) y Liau y Tan (2014), entre otros. De esta manera, las empresas de servicios que cuenten con empleados altamente capacitados en la gestión de los social media pueden utilizar una combinación integral de redes sociales que les permita lograr diferentes objetivos estratégicos, mientras que aquellas cuyos empleados estén menos capacitados, deberán elegir una o dos redes para conseguir sus objetivos organizativos (Valos et al., 2016). Además, tal y como recomienda Baird y Parisnis (2011), sería conveniente que las empresas formaran comunidades virtuales en las que se facilite el intercambio de ideas y conocimientos entre los empleados y se generen sinergias que permitan lanzar nuevas y mejores iniciativas al mercado.

Por último, también sería interesante que las empresas turísticas analizaran el impacto que las prácticas derivadas de este programa tienen sobre los resultados organizativos, tales como el nivel de lealtad del cliente, rentabilidad, niveles de calidad, reputación de las empresas, entre otros, al objeto de conocer si es conveniente invertir 
en la implementación de un programa de SCRM, tal y como señalan Diffley et al. (2018).

Por tanto, los resultados de la presente investigación, aunque no generalizables, aportan una visión muy valiosa que nos aproxima a conocer cómo se está enfrentando el tema de la gestión de las relaciones con los clientes en el sector turístico en Gran Canaria a través de los social media. De estos resultados se ha podido extraer la necesidad que perciben los directivos entrevistados de continuar manteniendo y gestionando los diversos social media en el largo plazo, con el fin de poder realizar análisis con los que recabar información continua y valiosa del cliente para la empresa. Asimismo, se puede extraer de los resultados que aún queda mucho camino por recorrer en este tema. Por otro lado, se ha puesto de manifiesto la inexistencia de análisis que aporten resultados tangibles que permitan conocer si existe una relación positiva entre su uso y la fidelización efectiva de los clientes. Para concluir y a modo de recomendación general, estas empresas deberían profundizar en este concepto como algo más que una mera herramienta tecnológica con la que recopilar información de sus clientes, sino ampliar su visión y orientación hacia una perspectiva estratégica de negocio que les lleve a conseguir una ventaja competitiva sostenible en el tiempo.

\section{LIMITACIONES Y FUTUROS ESTUDIOS}

Es preciso señalar que este trabajo no carece de limitaciones debido, entre otras, a su carácter exploratorio. En primer lugar, este tipo de investigación dota de cierta subjetividad a los resultados obtenidos, debido a la imposibilidad de interpretarlos de forma numérica o estadística y a la insuficiente representatividad de su muestra, que impiden su generalización. Sin embargo, estos inconvenientes no restan valor alguno a los resultados obtenidos en la investigación, gracias a la profundidad de las respuestas aportadas y a la riqueza de matices encontrados en las opiniones desinteresadas de los directivos entrevistados. Futuros trabajos de investigación deberían comenzar abordando este tema en otros contextos geográficos, esto es, ampliando el conocimiento del estado de la cuestión a otros destinos turísticos relevantes. Finalmente, sería útil incorporar la perspectiva de los clientes en próximos estudios, incluyendo aspectos relacionados con el efecto producido por la aplicación del SCRM en las empresas turísticas, tales como satisfacción, lealtad, compromiso, identificación y valor de marca.

\section{REFERENCIAS BIBLIOGRÁFICAS}

AGNIHOTRI, R., KOTHANDARAMAN, P., KASHYAP, R., \& SINGH, R. Bringing "social" into sales: The impact of salespeople's social media uses on service behaviors and value creation. En: Journal of Personal Selling and Sales Management. 2012, vol. 32, $\mathrm{n}^{\circ}$ 3, pp. 333 - 348.

ALMUNAWAR M. N., ANSHARI, M., y SUSANTO H. Crafting strategies for sustainability: how travel agents should react in facing a disintermediation. En: Operational Research, 2012, vol. 13, n 3, pp. 317-342.

BAGÓ, P. y VÖRÖS, P. Social Customer Relationship Management. En: Act Sci Soc. 2011, vol. 33, pp. 61-75.

BAIRD, C.H. y PARASNIS, G. From social media to Social CRM: reinventing the customer relationship. En: Strategy \& Leadership. 2011, vol. 39, nº 6, pp.27-34.

BERRY, L. L. y PARASURAMAN, A. Marketing Services. 1991. New York: Free Press. 
BOULDING, W., STAELIN, R., EHRET, M., y JOHNSTON, W. J. A customer relationship management roadmap: What is known, potential pitfalls, and where to go. En: Journal of Marketing. 2005, vol. 69, pp. 155-166.

BOYCE, C. y NEALE, P. A Guide for Designing and Conducting In-Depth Interviews for Evaluation Input. 2006. Pathfinder International Tool Series, Monitoring and Evaluation.

BRODIE, R.J., HOLLEBEEK, L., JURIC, B., y ILIC, A. Customer engagement: conceptual domain, fundamental propositions and implications for research. En: Journal of Service Research. 2011, vol. 14, n 3, pp. 1-20

BYGSTAD B. y PRESTHUS W. Social Media as CRM? How Two Scandinavian Airline Companies Used Facebook during the "Ash Crisis" in 2010. En: Scandinavian Journal of Information Systems. 2012, vol. 25, nº 1, pp. 51-72.

CAMBRA-FIERRO, J., CENTENO, E., OLAVARRÍA, A. y VÁZQUEZCARRASCO, R. Factores de éxito de CRM: un estudio exploratorio en el sector bancario español. En: Universia Business Review. 2014, vol. 43, pp. 144 - 167.

CARO HERRERO, J. L., LUQUE, A., y ZAYAS, B. Nuevas tecnologías para la interpretación y promoción de los recursos turísticos culturales. En: Pasos. Revista de Turismo y Patrimonio Cultural. 2015, vol. 13, nº 4, pp. 931-945.

CHAFFEY, D. E-business and E-commerce management (second edition). (2003). London: FT/Prentice Hall

CHAN, N. L., y DENIZCI GUILLET, B. Investigation of social media marketing: How does the hotel industry in Hong Kong perform in marketing on social media web-sites? En: Journal of Travel \& Tourism Marketing. 2011, vol. 28, no 4, pp. 345-368.

CHANG, J., YEN, D.C., KU, C-Y., y YOUNG D. Critical issues in CRM adoption and implementation. En: Int. J. Services Technology and Management. 2002, vol. 3, $\mathrm{n}^{\mathrm{o}} 3$, pp. 311-324.

CHATHOTH, P., ALTINAY, L., HARRINGTON, R.J., OKUMUS, F. y CHAN, E.S.W. Co-Production versus Co-Creation: A Process Based Continuum in the Hotel Service Context. En: International Journal of Hospitality Management. 2013, vol. 32, pp. 11-20

CHEN, H-M. y VARGO, S. L. Rethinking Social CRM Design: A Service Dominant Logic Perspective, In Handbook on e-business strategic management, Series PROGRESS in IS, editor Francisco J. Martínez-López, Springer, pp. 768-783, 2014

CHOUDHURY, M. M. y HARRIGAN, P., CRM to social CRM: the integration of new technologies into customer relationship management. En: Journal of Strategic Marketing. 2014, vol. 22, nº 2, pp. 149-176.

CHRISTOPHER, M., PAYNE, A. y BALLANTYNE, D. Marketing relacional. Integrando la calidad, el servicio al cliente y el marketing. 1994, Diaz de Santos, Madrid.

DIECK, M.C., JUNG, T.H., KIM, W. G., y MOON, Y. Hotel guests' social media acceptance in luxury hotels. En: International Journal of Contemporary Hospitality Management. 2017, vol. 29, nº 1, pp. 530-550. 
DIFFLEY, S., MCCOLE, P., y CARVAJAL-TRUJILLO, E. Examining social customer relationship management among Irish hotels. En: International Journal of Contemporary Hospitality Management. 2018, vol. 30, $\mathrm{n}^{\circ}$ 2, pp. 1072-1091.

DISTASIO, M. W., MCCORKINDALE, T., y WRIGHT, D. K. How public relations executives perceive and measure the impact of social media in their organizations. En: Public Relations Review. 2011, vol. 37, n 3, pp. 325-328.

EXCELTUR. Estudio de Impacto Económico del Turismo: IMPACTUR Canarias 2015. Disponible en: http://www.exceltur.org/wpcontent/uploads/2016/12/IMPACTUR-Canarias-2015.pdf

FAASE, R., R. HELMS y M. SPRUIT. Web 2.0 in CRM domain: defining Social CRM. En: Int. Journal of Electronic Customer Relationship Management. 2011, vol. $5, \mathrm{n}^{\mathrm{o}} 1$, pp. 1-22.

FJERMESTAD, J., y ROMANO, N. C. eCRM: General principles of usability \& resistance: Implementation framework. En: Business Process Management Journal. 2003, vol. 9, nº 5, pp. 572-591.

FROW, P. E., y PAYNE, A. F. Customer relationship management: a strategic perspective. En: Journal of Business Market Management. 2009, vol. 3, nº 1, pp. $7-27$.

GARRIDO-MORENO, A. y PADILLA-MELÉNDEZ, A. Estrategias CRM en empresas de servicios: recomendaciones directivas para su implementación. En: Dirección y Organización. 2012, vol. 46, pp. 56-66.

GARTNER. Social CRM Market Definition and Magic Quadrant Criteria. Analyst(s): Adam Sarner, Ed Thompson, Michael Maoz. Published: 05 February 2010 ID: G00174000

GREENBERG, P. The impact of CRM 2.0 on customer insight. En: Journal of Business \& Industrial Marketing. 2010, vol. 25, $\mathrm{n}^{\mathrm{o}}$ 6, pp. 410-419.

GRIFFIN, J. Customer Loyalty: How to Earn It, How to Keep It, New and Revised Edition, Weekly publisher, 2002.

GRÖNROOS, C. Relationship marketing: The strategy continuum. En: Journal of the Academy of Marketing Science. 1995, vol. 23, n 4, pp. 252

HACKNEY, D. CRM prerequisite \#1: A data warehouse system. En: Business and Management Practices. 2000, September, vol. 10, nº 9, pp. 42.

HANNA, R., ROHM, A., y CRITTENDEN, V. L. We're all connected: the power of the social media Ecosystem. En: Business Horizons. 2011, vol. 54, no 3, pp. 265273.

HARRIGAN, P., EVERS, U., MILES, M., y DALY, T. (2017). Customer engagement with tourism social media brands. En: Tourism Management, vol. 59, pp. 597609.

HARRIGAN, P. y MILES, M. From e-CRM to s-CRM. Critical factors underpinning the social CRM activities of SMEs. En: Small Enterprise Research. 2014, vol. 21, nº 1, pp. 99-116.

HASANI, T., BOJEI, J., y DEHGHANTANHA, A. Investigating the antecedents to the adoption of SCRM technologies by start-up companies. En: Telemat Inform. 2017, vol. 5, no 34, pp. 655-675. 
HENNIG-THURAU, T., GWINNER, K.P. y GREMLER, D.D. Understanding relationship marketing outcomes: an integration of relational benefits and relationship quality. En: Journal of Service Research. 2002, vol. 4, nº 3, pp. 230247.

HOLLEBEEK, L., GLYNN, M. S., y BRODIE, R. J. Consumer brand engagement in social media: conceptualization, scale development and validation. En: Journal of Interactive Marketing. 2014, vol. 28, $\mathrm{n}^{\mathrm{o}}$ 2, pp. 149-165.

HOSTELTUR. Tendencias 2017 en Social Media para destinos turísticos aventajados. Disponible en: https://www.hosteltur.com/120002_tendencias-2017-socialmedia-destinos-turisticos-aventajados.html

KANDAMPULLY, J. y SUHARTANTO, D. Customer loyalty in the hotel industry: the role of customer satisfaction and image. En: International Journal of Contemporary Hospitality Management. 2000, vol. 12, nº 6, pp.346-351,

KAPLAN, A. M. y HAENLEIN, M. Users of the world, unite! The challenges and opportunities of Social Media. En: Business Horizons. 2010, vol. 53, $\mathrm{n}^{\mathrm{o}}$ 1, pp. 59-68.

KIM, J., SUH, E. y HWANG, H. A model for evaluating the effectiveness of CRM using the balanced scorecard. En: Journal of Interactive Marketing. 2003, vol. $17, \mathrm{n}^{\mathrm{o}} 2$, pp. 5-19.

KING, S. F., y BURGESS T. F. Understanding success and failure in customer relationship management. En: Industrial Marketing Management. 2008, vol. 37, $\mathrm{n}^{\mathrm{o}}$ 4, pp. 421-431.

LEUNG, D., LAW, R., VAN HOOF, H., y BUHALIS, D. Social media in tourism and hospitality: a literature review. En: Journal of Travel \& Tourism Marketing, 2013, vol. 30, pp. 3-22.

LI, C.H. y CHANG, C.M. The influence of trust and perceived playfulness on the relationship commitment of hospitality online social network - moderating effects of gender. En: International Journal of Contemporary Hospitality Management. 2016, vol. 28, $\mathrm{n}^{\circ}$ 5, pp. 924-944.

LI, X. y WANG, Y. C. China in the eyes of west-ern travelers as represented in travel blogs. En: Journal of Travel \& Tourism Marketing. 2011, vol. 28, nº 7, pp. 689719

LIAO, S.H., CHEN, Y.J. y DENG, M.Y. Mining customer knowledge for tourism new product development and customer relationship management. En: Expert Systems with Applications. 2010, vol. 37, pp. 4212-4223.

LIAU, B.Y. y TAN, P.P. Gaining customer knowledge in low cost airlines through text mining. En: Industrial Management \& Data Systems. 2014, vol. 114, n 9, pp. 1344-1359.

LO, A.S., IM, H. H., CHEN, Y. y QU, H. Building brand relationship quality among hotel loyalty program members. En: International Journal of Contemporary Hospitality Management. 2017, vol. 29, n 1, pp. 458-488.

MAGGON M. y CHAUDHRY H. Revisiting Relationship Marketing and Customer Relationship Management in Leading Tourism and Hospitality Journals: Research Trends From 2001 to 2013, En: Journal of Relationship Marketing. 2015, vol. $14, \mathrm{n}^{\mathrm{o}} 1$, pp. 53-77. 
MOHAN, S., CHOI, E., y MIN, D. Conceptual modeling of enterprise application system using social networking and Web 2.0 social CRM system. Proceedings of International Conference on Convergence and Hybrid Information Technology (ICHT) in Daejeon, South Korea, 2008, IEEE, pp. 237-244.

MUNNUKKA, J., y JÄRVI, P. Perceived risks and risk Management of Social Media in an organizational context. En: Electronic Markets. 2014, vol. 24, n 3, pp. 219229.

NGUYEN, T. H., SHERIF, J.S., y NEWBY M. Strategies for successful CRM implementation. En: Information Management \& Computer Security. 2007, vol. $15, \mathrm{n}^{\mathrm{o}} 2$, pp. 102-115.

NOONE, B. N., MCGUIRE, K. A., y ROHLFS, K. V. Social media meets hotel revenue manage-ment: Opportunities, issues and unanswered questions. En: Journal of Revenue and Pricing Management. 2011, vol. 10, n 4, pp. 293-305.

ÖZGENER, S. y IRAZ, R. Customer relationship management in small-medium enterprises: The case of Turkish tourism industry. En: Tourism Management. 2006, vol. 27, nº 6 , pp. 1356-1363.

PALAZÓN, M., SICILIA, M. y DELGADO, E. El papel de las redes sociales como generadoras de "amor a la marca". En: Universia Business Review. 2014 vol. 41, pp. 18-39.

PATRONATO DE TURISMO Situación del sector turístico, Gran Canaria. (2015). Disponible en: http://www.grancanaria.com/patronato_turismo/typo3conf/ext/naw_securedl/sec ure.php? $\mathrm{u}=0 \&$ file=fileadmin/PDF/informes/ICT_abril_15.pdf\&t=1434900124\& hash=3c4ae62969c67fe6a3bd826815293b5c

PROMOTUR TURISMO CANARIAS. Islas Canarias, Quinto destino turístico en redes sociales para los británicos. Disponible en http://turismodeislascanarias.com/es/actualidad/islas-canarias-quinto-destinoturistico-en-redes-sociales-para-los-britanicos/ (12/06/2017)

RAPP, A. y OGILVIE, J. CRM and social media, in Nguyen, B., Simkin, L. and Canhoto, A.I. (Eds), The Dark Side of CRM: Customers, Relationships and Management, Routledge, 2016, Oxon, pp. 58-73

REINARTZ, W., KRAFFT, M., y HOYER, W. The Customer Relationship Management Process: Its Measurement and Impact on Performance. En: Journal of Marketing Research. 2004, vol. 12, pp. 293-305.

REINHOLD, O., y ALT, R. Analytical Social CRM: Concept and Tool Support. BLED 2011 Proceedings, pp. 226-241.

RODRIGUEZ, M., PETERSON, R. M., y KRISHNAN, V. Social media's influence on business-to business sales performance. En: Journal of Personal Selling \& Sales Management. 2012, vol. 32, pp. 365-378.

ROSALES, P. Estrategia digital. Cómo usar las nuevas tecnologías mejor que la competencia. Barcelona: Deusto, 2010.

ROSMAN R. y STUHURA, K. The Implications of Social Media on Customer Relationship Management and the Hospitality Industry. En: Journal of Management Policy and Practice. 2013, vol. 14, nº 3, pp. 18-26. 
SALKHORDEH, P. Key issues in use of social net-working in hospitality industry: 2009. Retrieved from http://scholarworks.umass.edu/cgi/viewcontent.cgi?article $=1259 \&$ context=gradconf_hospitality

SCULLIN, S., FJERMESTAD, J., y ROMANO, N. C. E-relationships marketing: Changes in traditional marketing as the outcome of eCRM. En: Journal of Enterprise Information Management. 2004, vol. 17, nº 6, pp. 410-415.

SIGALA, M. Integrating customer relationship management in hotel operations: Managerial and operational implications. En: Hospitality Management. 2005, vol. 24, no 3, pp. 391-413.

SIGALA, M. ECRM 2.0 applications and trends: The use and perceptions of Greek tourism firms of social networks and intelligence. En: Computers in Human Behavior. 2011, vol. 27, n 2, pp. 655-661.

SIGALA M. Social CRM Capabilities and Readiness: Findings from Greek Tourism Firms. In: Inversini A., Schegg R. (eds) Information and Communication Technologies in Tourism 2016. Springer, Cham

TEO, T.S.H., DEVADOSS, P., y PAN, S. L. Towards a holistic perspective of customer relationship management (CRM) implementation: A case study of the Housing and Development Board, Singapore. En: Decision Support Systems. 2006, vol.42, pp. 1613-1627

TRAINOR, K. J. Relating social media technologies to performance: A capabilitiesbased perspective. En: Journal of Personal Selling \& Sales Management. 2012, vol. 32, no 3, pp. 317-331.

THRYAMBAKAM, P. y BETHAPUDI, A. Customer Relationship Management Challenges in Hospitality and Tourism Faced by Various Stakeholders in Andhra Pradesh. En: Global Journal of Management and Business Studies. 2013, vol. 3, nº 11, pp. 1261-1268.

TRAINOR, K. J., ANDZULIS, J. M., RAPP, A., y AGNIHOTRI, R. Social media technology usage and customer relationship performance: a capabilities-based examination of social CRM. En: Journal of Business Research. 2014, vol. 67, $\mathrm{n}^{\mathrm{o}}$ 6, pp. 1201-1208.

TSIMONIS, G. y DIMITRIADIS, S. Brand strategies in social media. En: Mark Intell Plan. 2014, vol. 32, pp. 3328-3344.

VALOS M. J., HABIBI F. H., CASIDY R., DRIESENER C. B., y MAPLESTONE V. L. Exploring the inte-gration of social media within integrated marketing communication frameworks, En: Marketing Intelligence \& Planning. 2016, vol. 34, no. 1, pp. 19-40.

VAN DOORN J, LEMON KE, MITTAL V, NAB S, PICK D, PIRNER P, ET AL. Customer engagement behavior: theoretical foundations and research directions. En: Journal of Service Research, 2010, vol. 13, n 3, pp. 253-66.

VAN LOOY, A. Social Media Management: Technologies and Strategies for Creating Business Value. 2016. NY: Springer.

WINER, R., A framework for customer relationship management. En: California Management Review. 2001, vol. 43, n 4, pp. 89-106. 
WOOD, M. Changing the rules of enterprise data warehousing. En: Health Management Technology. 2000, vol. 21, nº 9, pp. 56.

WOODCOCK, N., GREEN, A., y STARKEY, M. Social CRM as a business strategy. En: Journal of Database Marketing \& Customer Strategy Management. 2011, vol. $18, \mathrm{n}^{\mathrm{o}} 1$, pp. $50-64$.

WORLD ECONOMIC FORUM. The Travel \& Tourism Competitiveness Report 2017. Paving the Way for a More Sustainable and Inclusive Future Eds. Crotti, R. y Misrahi, T., Ginebra, Suiza

XIANG, Z., y GRETZEL, U. Role of social media in online travel information search. En: Tourism Management. 2010, vol. 31, $\mathrm{n}^{\circ}$ 2, pp. 179-188.

YEN H. J., y GWINNER, K.P. Internet retail customer loyalty: the mediating role of relational benefits. En: International Journal of Service Industry Management. 2003, vol. 14, no 5, pp. 483-500.

YOON, K., y SIMS, J. D. Integrating social media and traditional CRM: Toward a conceptual framework for social CRM practices. In A. Ayanso, \& K. Lertwachara (Eds.), Harnessing the power of social media and web analytics: Techniques, tools, and applications. 2013. Academic Press.

ZALIZNYAK, M. La cara más social del CRM. En: Harvard Deusto. 2013, vol. 116, pp. 70-76.

ZENG, B. y GERRITSEN, R. What do we know about social media in tourism? A review. En: Tourism Management Perspectives. 2014, vol. 10, pp. 27-36. 